\title{
含反应扩散项的时滞细胞神经网络模型的 $W^{1,2}(\Omega)$ - 与 $X^{1,2}(\Omega)$-稳定性
}

罗毅平 (1)*, 夏文华 (2), 刘国荣 (1), 邓飞其 ${ }^{3}$

(1) 湖南工程学院智能控制与智能信息处理研究所, 湘潭 411101;

(2) 湖南工程学院理学院, 湘潭 411101 ;

(3) 华南理工大学系统工程研究所, 广州 510640

*通信作者 E-mail: lyp8688@sohu.com

收稿日期: 2008-04-12；接受日期: 2008-12-05

国家自然科学基金 (批准号: 60374023)、湖南省自然科学基金 (批准号: 07JJ6112) 和湖南省教育厅重点 (批准号: 04A012, 07A015) 资助 项目

摘要针对一类具反应扩散项的时滞细胞神经网络模型, 利用 Poincare 不等式与辅 助函数的方法, 获得了该系统的 $W^{1,2}(\Omega)$-指数稳定性和 $X^{1,2}(\Omega)$-渐近稳定性条件; 与 以往文献结论不同, 这些稳定性条件包含了扩散算子项, 与以往结果比较, 获得的指数 稳定性条件更强. 最后, 通过实例说明该方法的可行性.

\section{关键词}

细胞神经网络

反应扩散

$W^{1,2}(\Omega)$-指数稳定性 $X^{1,2}(\Omega)$-渐近稳定性 时滞

\section{1 引言}

众所周知, 神经网络稳定性是神经网络模型应用 于实际的前提条件, 由于神经网络在图像传输、信 号传递、最优计算等方面广泛的及潜在的应用性, 使得对神经网络的动力学特性的研究吸引了许多学 者. 而图像的传输等存在着时滞, 故现在有许多文 献主要研究时滞神经网络的动力学性质 $[1 \sim 11]$. 当 考虑电子在一个非均匀的电磁场中运行时, 反应扩 散现象是不可避免的, 也是不可忽视的 ${ }^{[12,13]}$. 因此 现在有一些研究者研究了具反应扩散的神经网络模

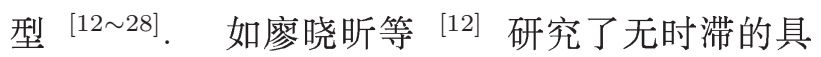
反应扩散的神经网络模型, 而王林山等 ${ }^{[13]}$ 、 Liang 等 ${ }^{[14]}$ 、Son 等 ${ }^{[15]}$ 研究了含时滞的具反应扩散项的 神经网络模型. 他们都是利用 Halanay 不等式或推 广的 Halanay 不等式获得稳定性条件. 在利用散度 定理后, 最后都去掉了一个负的含梯度的积分项. 这 样导致他们所获得的稳定性条件中不含有扩散算子
项, 这就是说扩散算子项在他们的稳定性条件中没 起到作用. 他们获得的稳定性条件与不含反应扩散 项的神经网络模型的稳定性条件是一样的, 此外, 文 献 [16 28] 的结果也存在同样的的情况. 下面我们提 出一种新的方法, 利用 Poincare 不等式与辅助函数 等知识获得一个更强的指数稳定性条件, 同时, 我们 的稳定性条件中将包含扩散算子对系统的影响.

\section{2 具反应扩散项时滞神经网络模型的稳定性}

我们引入下面的引理:

引理 $\mathbf{1}^{[29]}$ 设 $U_{1}, U_{2}, U_{3}$ 是一定维数的实矩阵, 且有 $U_{3}=U_{3}^{\mathrm{T}}>0$, 则任给标量 $\beta>0$, 使得下列不 等式成立:

$$
U_{2}^{\mathrm{T}} U_{1}+U_{1}^{\mathrm{T}} U_{2} \leqslant \beta^{-1} U_{1}^{\mathrm{T}} U_{3}^{-1} U_{1}+\beta U_{2}^{\mathrm{T}} U_{3} U_{2} .
$$

\section{1 常时滞系统的稳定性}

考虑含反应扩散项的细胞神经网络模型

$$
\frac{\partial \bar{u}_{i}}{\partial t}=\sum_{k=1}^{m} \frac{\partial}{\partial x_{k}}\left(D_{i k} \frac{\partial \bar{u}_{i}}{\partial x_{k}}\right)
$$




$$
\begin{aligned}
& -c_{i} \bar{u}_{i}(x, t)+\sum_{j=1}^{n} a_{i j} \tilde{f}_{j}\left(\bar{u}_{j}(x, t)\right) \\
& +\sum_{j=1}^{n} b_{i j} \tilde{f}_{j}\left(\bar{u}_{j}(x, t-\tau)\right)+I_{i}, \\
& x \in \Omega, \quad i=1, \ldots, n .
\end{aligned}
$$

满足初边值条件

$$
\begin{gathered}
\frac{\partial \bar{u}_{i}}{\partial n}=0, \quad t \geqslant 0, \\
x \in \partial \Omega, \quad i=1, \ldots, n . \\
\bar{u}_{i}(x, s)=\bar{\varphi}_{i}(x, s),-\tau \leqslant s \leqslant 0, \\
i=1, \ldots, n .
\end{gathered}
$$

其中光滑函数 $D_{i k}=D_{i k}(x, t, u) \geqslant 0$ 表示扩散算子. $\tau$ 表示轴突信号传输过程中的延迟. $c_{i}>0$ 表示在与 神经网络不连通并且无外部附加电压差的情况下第 $i$ 个神经元恢复孤立静息状态下的速率. $a_{i j}$ 表示神 经元之间相互.联络的权. $u_{i}, x_{i}$ 分别表示状态变量和 空间变量. $I_{i}$ 表示外部输入. $f_{j}$ 为激活函数.

$$
\frac{\partial u_{i}}{\partial n}=0, \quad t \geqslant 0, x \in \partial \Omega,
$$

$\phi_{i}(s, x)$ 是初值和边值. $\Omega=\left\{x=\left(x_{1}, \ldots, x_{m}\right),\left|x_{i}\right|<\right.$ $l\}$ 是具有光滑边界的紧集, 并且在 $R^{m}$ 中的测度 $\operatorname{mes} \Omega>0$.

假设模型 $(1)$ 满足下面的假设条件

$(\mathrm{H} 1)$ 假设激活函数 $\tilde{f}_{j}(\cdot)(j=1, \ldots, n)$ 满足

$$
0 \leqslant \frac{\tilde{f}_{j}(x)-\tilde{f}_{j}(y)}{x-y} \leqslant l_{j}
$$

对任意的 $x, y \in \mathbb{R}$.

设 $u^{*}=\operatorname{col}\left(u_{1}^{*}, \ldots, u_{n}^{*}\right)$ 是模型 $(1)$ 的一个平衡 点. 则 $u^{*}$ 满足下列方程:

$$
\begin{aligned}
& -c_{i} u_{i}^{*}+\sum_{j=1}^{n} a_{i j} \tilde{f}_{j}\left(u_{j}^{*}\right)+\sum_{j=1}^{n} b_{i j} \tilde{f}_{j}\left(u_{j}^{*}\right)+I_{i}=0, \\
& \quad i=1, \ldots, n .
\end{aligned}
$$

则模型 (1) 的第一式可化为

$$
\begin{aligned}
\frac{\partial\left(\bar{u}_{i}-u_{i}^{*}\right)}{\partial t}= & \sum_{k=1}^{m} \frac{\partial}{\partial x_{k}}\left(D_{i k} \frac{\partial\left(\bar{u}_{i}-u_{i}^{*}\right)}{\partial x_{k}}\right) \\
& -c_{i}\left(\bar{u}_{i}(x, t)-u_{i}^{*}\right)+\sum_{j=1}^{n} a_{i j}\left(\tilde{f}_{j}\left(\bar{u}_{j}(x, t)\right)\right. \\
& \left.-\tilde{f}_{j}\left(u_{j}^{*}\right)\right)+\sum_{j=1}^{n} b_{i j}\left(\tilde{f}_{j}\left(\bar{u}_{j}(x, t-\tau)\right)\right. \\
& \left.-\tilde{f}_{j}\left(u_{j}^{*}\right)\right), \quad i=1, \ldots, n
\end{aligned}
$$

令 $\bar{u}_{i}-u_{i}^{*}=u_{i}, \tilde{f}_{j}\left(\bar{u}_{j}(x, t)\right)-\tilde{f}_{j}\left(u_{j}^{*}\right)=f_{j}\left(u_{j}(x, t)\right)$.

则 (4) 式可化为

$$
\begin{aligned}
\frac{\partial u_{i}}{\partial t}= & \sum_{k=1}^{m} \frac{\partial}{\partial x_{k}}\left(D_{i k} \frac{\partial u_{i}}{\partial x_{k}}\right) \\
& -c_{i} u_{i}+\sum_{j=1}^{n} a_{i j} f_{j}\left(u_{j}(x, t)\right) \\
& +\sum_{j=1}^{n} b_{i j} f_{j}\left(u_{j}(x, t-\tau), \quad i=1, \ldots, n .\right.
\end{aligned}
$$

写成矩阵矩阵形式即为:

$$
\begin{aligned}
\frac{\partial u}{\partial t}= & D \Delta u(x, t)-C u(x, t)+A f(u(x, t)) \\
& +B f(u(x, t-\tau) .
\end{aligned}
$$

记 $L^{2}(\Omega)$ 是 $\Omega$ 上的实 Lebesgue 可测函数空间 且对于 $L_{2^{-}}$模

$$
\|u\|_{L^{2}}=\left[\int_{\Omega}|u(x)|^{2} \mathrm{~d} x\right]^{1 / 2}
$$

构成一个 Banach 空间. 其中 $|u|$ 表示向量 $u \in \mathbb{R}^{n}$ 的 Euclid 模, 并称为 E-模.

我们引入下面的稳定性定义.

定义 $\mathbf{1}^{[30]}$ 若存在正数 $\eta$ 和 $\varepsilon$, 使得

$$
\begin{gathered}
\|u(x, t)\|_{L^{2}}^{2} \leqslant \varepsilon \sup _{t_{0}-\tau \leqslant s \leqslant t_{0}}\left[\|u(x, \mathrm{~s})\|_{L^{2}}^{2}\right] e^{-\eta t}, \\
(x, t) \in \Omega \times R_{+},
\end{gathered}
$$

则称系统 $(1)$ 是 $L^{2}(\Omega)$ - 指数渐近稳定的.

定义 $2^{[30]}$ 若系统 $(1)$ 是 $L^{2}(\Omega)$-指数渐近稳定 的. 并且存在正数 $\theta$, 使得

$$
\lim _{t \rightarrow \infty}\|\nabla u(x, t)\|_{L^{2}}^{2} e^{\theta \tau}=0, \quad x \in \Omega
$$

则称系统 $(1)$ 是 $W^{1,2}(\Omega)$-指数渐近稳定的.

定理 1 对于系统 (1), 若存在正定对称矩阵 $P_{1}, P_{2}$ 与正数 $\varepsilon, \alpha$, 并选取 $\gamma=\alpha\left\|L^{\mathrm{T}} P_{2}^{-1} L\right\|$, 使得

(i) $(2 \tilde{D}-\varepsilon I)>0$,

(ii) $\lambda=-\lambda_{\max }\left(-2 C+B P_{2} B^{\mathrm{T}}+A P_{1} A^{\mathrm{T}}\right)+\frac{m}{l^{2}}$. $\lambda_{\min }(2 \tilde{D}-\varepsilon I)>0$,

(iii) $\lambda>\frac{\gamma}{\alpha}$,

其中 $\tilde{D}=\operatorname{diag}\left(D_{1}, \ldots, D_{n}\right), D_{i}=\min _{1 \leqslant k \leqslant m}\left\{D_{i k}\right\}$, $i=1, \ldots, n$. 则有系统 $(1)$ 是全局 $W^{1,2}(\Omega)$-指数渐近 稳定的.

\section{证明 作辅助函数}

$$
v(x, t)=\alpha u^{\mathrm{T}}(x, t) u(x, t)+\gamma \int_{t-\tau}^{t} u^{\mathrm{T}}(x, s) u(x, s) \mathrm{d} s .
$$


两边对 $t$ 求导得:

$$
\begin{aligned}
\frac{\partial v(x, t)}{\partial t}= & 2 \alpha u^{\mathrm{T}}(x, t)[D \Delta u(x, t)-C u(x, t) \\
& +A f(u(x, t))+B f(u(x, t-\tau)] \\
& +\gamma u^{\mathrm{T}}(x, t) u(x, t) \\
& -\gamma u^{\mathrm{T}}(x, t-\tau) u(x, t-\tau) .
\end{aligned}
$$

对 (8) 式两边在区域 $\Omega$ 上对 $x$ 积分, 则有

$$
\begin{aligned}
& \frac{\mathrm{d}}{\mathrm{d} t}\left[\int_{\Omega} v(x, t) \mathrm{d} x\right] \\
& =2 \alpha \int_{\Omega} u^{\mathrm{T}}(x, t) D \Delta u(x, t) \mathrm{d} x \\
& \quad-2 \alpha \int_{\Omega} u^{\mathrm{T}}(x, t) C u(x, t) \mathrm{d} x \\
& \quad+2 \alpha \int_{\Omega} u^{\mathrm{T}}(x, t) A f(u(x, t)) \mathrm{d} x \\
& \quad+2 \alpha \int_{\Omega} u^{\mathrm{T}}(x, t) B f(u(x, t-\tau)) \mathrm{d} x \\
& \left.\quad+\gamma \int_{\Omega} u^{\mathrm{T}}(x, t) u(x, t)\right] \mathrm{d} x \\
& \quad-\gamma \int_{\Omega} u^{\mathrm{T}}(x, t-\tau) u(x, t-\tau) \mathrm{d} x .
\end{aligned}
$$

注意到散度定理和边值条件

$$
\begin{aligned}
& \int_{\Omega}\left[\nabla(u(x, t))^{\mathrm{T}} D \nabla(u(x, t))\right. \\
& \left.\quad+(u(x, t))^{\mathrm{T}} D \Delta(u(x, t))\right] \mathrm{d} x \\
& =\int_{\partial \Omega}(u(x, t))^{\mathrm{T}} D \frac{\partial(u(x, t))}{\partial t} \mathrm{~d} x=0 .
\end{aligned}
$$

于是由 (9) 式可得

$$
\begin{aligned}
& \frac{\mathrm{d}}{\mathrm{d} t}\left[\int_{\Omega} v(x, t) \mathrm{d} x\right] \\
& \leqslant-2 \alpha \int_{\Omega} \nabla(u(x, t))^{\mathrm{T}} D \nabla(u(x, t)) \mathrm{d} x \\
& \quad+\alpha \int_{\Omega} u^{\mathrm{T}}(x, t)\left[-2 C+B P_{2} B^{\mathrm{T}}\right. \\
& \left.\quad+A P_{1} A^{\mathrm{T}}+L^{\mathrm{T}} P_{1}^{-1} L+\frac{\gamma}{\alpha} I\right] u(x, t) \mathrm{d} x \\
& \quad-\gamma \int_{\Omega} u^{\mathrm{T}}(x, t-\tau) u(x, t-\tau) \mathrm{d} x \\
& \quad+\alpha \int_{\Omega} u^{\mathrm{T}}(x, t-\tau) L^{\mathrm{T}} P_{2}^{-1} L u(x, t-\tau) \mathrm{d} x .
\end{aligned}
$$

令 $\theta=\frac{\gamma}{\alpha}$, 则

$$
\begin{aligned}
& \frac{\mathrm{d}}{\mathrm{d} t}\left[\int_{\Omega} v(x, t) \mathrm{d} x\right] \\
& \leqslant-2 \alpha \int_{\Omega} \nabla(u(x, t))^{\mathrm{T}} D \nabla(u(x, t)) \mathrm{d} x
\end{aligned}
$$

$$
\begin{aligned}
& +\alpha \int_{\Omega} u^{\mathrm{T}}(x, t)\left[-2 C+B P_{2} B^{\mathrm{T}}\right. \\
& \left.+A P_{1} A^{\mathrm{T}}+L^{\mathrm{T}} P_{1}^{-1} L+\theta I\right] u(x, t) \mathrm{d} x .
\end{aligned}
$$

\section{又直接由 (7) 式可得}

$$
\begin{aligned}
& \frac{\mathrm{d}}{\mathrm{d} t}\left[\int_{\Omega} v(x, t) \mathrm{d} x\right] \\
& =\alpha \frac{\mathrm{d}}{\mathrm{d} t} \int_{\Omega} u(x, t)^{\mathrm{T}} u(x, t) \mathrm{d} x \\
& \quad+\gamma \int_{\Omega} u^{\mathrm{T}}(x, t) u(x, t) \mathrm{d} x \\
& \quad-\gamma \int_{\Omega} u^{\mathrm{T}}(x, t-\tau) u(x, t-\tau) \mathrm{d} x .
\end{aligned}
$$

从而

$$
\begin{aligned}
\frac{\mathrm{d}}{\mathrm{d} t} \int_{\Omega} u(x, t)^{\mathrm{T}} u(x, t) \mathrm{d} x & \frac{1}{\alpha} \frac{\mathrm{d}}{\mathrm{d} t}\left[\int_{\Omega} v(x, t) \mathrm{d} x\right] \\
& -\gamma \int_{\Omega} u^{\mathrm{T}}(x, t) u(x, t) \mathrm{d} x \\
& +\gamma \int_{\Omega} u^{\mathrm{T}}(x, t-\tau) u(x, t-\tau) \mathrm{d} x \\
\leqslant & -2 \int_{\Omega} \nabla(u(x, t))^{\mathrm{T}} D \nabla(u(x, t)) \mathrm{d} x \\
& +\int_{\Omega} u^{\mathrm{T}}(x, t)\left[-2 C+B P_{2} B^{\mathrm{T}}+A P_{1} A^{\mathrm{T}}\right. \\
& \left.+L^{\mathrm{T}} P_{1}^{-1} L\right] u(x, t) \mathrm{d} x \\
& +\theta \int_{\Omega} u^{\mathrm{T}}(x, t-\tau(t)) u(x, t-\tau(t)) \mathrm{d} x .
\end{aligned}
$$

注意到 Poincare 不等式 ${ }^{[31]}$, 我们有

$$
\begin{aligned}
& \int_{\Omega} u(x, t)^{\mathrm{T}} u(x, t) \mathrm{d} x \\
& =\sum_{i=1}^{n} \int_{\Omega} u_{i}^{2}(x, t) \mathrm{d} x \\
& \leqslant \frac{l^{2}}{m} \sum_{i=1}^{n} \int_{\Omega}\left|\nabla u_{i}(x, t)\right|^{2} \mathrm{~d} x \\
& =\frac{l^{2}}{m} \int_{\Omega} \nabla(u(x, t))^{\mathrm{T}} \nabla(u(x, t)) \mathrm{d} x .
\end{aligned}
$$

又

$$
\begin{aligned}
& 2 \int_{\Omega} \nabla(u(x, t))^{\mathrm{T}} D \nabla(u(x, t)) \mathrm{d} x \\
& \leqslant 2 \int_{\Omega} \nabla(u(x, t))^{\mathrm{T}} \tilde{D} \nabla(u(x, t)) \mathrm{d} x \\
& =\varepsilon \int_{\Omega} \nabla(u(x, t))^{\mathrm{T}} \nabla(u(x, t)) \mathrm{d} x \\
& \quad+\int_{\Omega} \nabla(u(x, t))^{\mathrm{T}}(2 \tilde{D}-\varepsilon I) \nabla(u(x, t)) \mathrm{d} x
\end{aligned}
$$




$$
\begin{aligned}
\geqslant & \varepsilon \int_{\Omega} \nabla(u(x, t))^{\mathrm{T}} \nabla(u(x, t)) \mathrm{d} x \\
& +\lambda_{\min }(2 \tilde{D}-\varepsilon I) \cdot \frac{m}{l^{2}} \int_{\Omega} u(x, t)^{\mathrm{T}} u(x, t) \mathrm{d} x .(16)
\end{aligned}
$$

于是, 由 (14) (16) 式可得

$$
\begin{aligned}
\frac{\mathrm{d}}{\mathrm{d} t} & \int_{\Omega} u(x, t)^{\mathrm{T}} u(x, t) \mathrm{d} x \\
\leqslant & -\varepsilon \int_{\Omega} \nabla(u(x, t))^{\mathrm{T}} \nabla(u(x, t)) \mathrm{d} x \\
& -\lambda_{\min }(2 \tilde{D}-\varepsilon I) \cdot \frac{m}{l^{2}} \int_{\Omega} u(x, t)^{\mathrm{T}} u(x, t) \mathrm{d} x \\
& +\int_{\Omega} u^{\mathrm{T}}(x, t)\left[-2 C+B P_{2} B^{\mathrm{T}}\right. \\
& \left.+A P_{1} A^{\mathrm{T}}+L^{\mathrm{T}} P_{1}^{-1} L\right] u(x, t) \mathrm{d} x \\
& +\theta \int_{\Omega} u^{\mathrm{T}}(x, t-\tau) u(x, t-\tau) \mathrm{d} x \\
\leqslant & -\varepsilon \int_{\Omega} \nabla(u(x, t))^{\mathrm{T}} \nabla(u(x, t)) \mathrm{d} x \\
& -\lambda_{\min }(2 \tilde{D}-\varepsilon I) \cdot \frac{m}{l^{2}} \int_{\Omega} u(x, t)^{\mathrm{T}} u(x, t) \mathrm{d} x \\
& +\lambda_{\max }\left(-2 C+B P_{2} B^{\mathrm{T}}+A P_{1} A^{\mathrm{T}}\right. \\
& \left.+L^{\mathrm{T}} P_{1}^{-1} L\right) \int_{\Omega} u^{\mathrm{T}}(x, t) u(x, t) \mathrm{d} x \\
& +\theta \int_{\Omega} u^{\mathrm{T}}(x, t-\tau) u(x, t-\tau) \mathrm{d} x \\
\leqslant & -\varepsilon \int_{\Omega} \nabla(u(x, t))^{\mathrm{T}} \nabla(u(x, t)) \mathrm{d} x \\
& -\lambda \int_{\Omega} u(x, t)^{\mathrm{T}} u(x, t) \mathrm{d} x \\
& +\theta \int_{\Omega} u^{\mathrm{T}}(x, t-\tau) u(x, t-\tau) \mathrm{d} x . \\
&
\end{aligned}
$$

由此可得

$$
\begin{aligned}
& \frac{\mathrm{d}}{\mathrm{d} t}\left(\|u(x, t)\|_{L^{2}}^{2}\right) \\
& \leqslant-\varepsilon\|\nabla(u(x, t))\|_{L^{2}}^{2} \\
& \quad-\lambda\|u(x, t)\|_{L^{2}}^{2}+\theta\|u(x, t-\tau)\|_{L^{2}}^{2} .
\end{aligned}
$$

积分上式得

$$
\begin{aligned}
\|u(x, t)\|_{L^{2}}^{2} \leqslant & \|u(x, 0)\| e^{-\lambda t} \\
& -\varepsilon \int_{0}^{t}\|\nabla(u(x, s))\|_{L^{2}}^{2} e^{-\lambda(t-s)} \mathrm{d} s \\
& +\theta \int_{0}^{t}\|u(x, s-\tau)\|_{L^{2}}^{2} e^{-\lambda(t-s)} \mathrm{d} s .
\end{aligned}
$$

再作辅助函数

$$
f(y)=1-\frac{\theta e^{y \tau}}{\lambda-y}
$$

因为 $f(0)=1-\frac{\theta}{\lambda}>0$, 于是存在一个整数 $b$, 使得 $f(y)$ 在区间 $[0, b)$ 上连续, 并且 $0<f(y)<1$, 取充分 小的 $\delta \in(0, b)$, 使得 $\lambda-\delta>0$, 且 $f(\delta)>0$. 对此 $\delta$, 用 $e^{\delta t}$ 乘 (19) 两边, 可得

$$
\begin{aligned}
\|u(x, t)\|_{L^{2}}^{2} e^{\delta t} & \|u(x, 0)\| e^{-(\lambda-\delta) t} \\
& -\varepsilon \int_{0}^{t}\|\nabla(u(x, s))\|_{L^{2}}^{2} e^{-(\lambda-\delta)(t-s)} e^{\delta s} \mathrm{~d} s \\
& +\theta e^{\delta \tau} \int_{0}^{t}\|u(x, s-\tau)\|_{L^{2}}^{2} e^{-(\lambda-\delta)(t-s)} e^{\delta(s-\tau)} \mathrm{d} s \\
\leqslant & \|u(x, 0)\| e^{-(\lambda-\delta) t} \\
& -\varepsilon \int_{0}^{t}\|\nabla(u(x, s))\|_{L^{2}}^{2} e^{-(\lambda-\delta)(t-s)} e^{\delta s} \mathrm{~d} s \\
& +\theta e^{\delta \tau} \int_{0}^{t}\|u(x, s-\tau)\|_{L^{2}}^{2} e^{-(\lambda-\delta)(t-s)} e^{\delta s} \mathrm{~d} s \\
\leqslant & \|u(x, 0)\|-\varepsilon \int_{0}^{t}\|\nabla(u(x, s))\|_{L^{2}}^{2} \\
& e^{-(\lambda-\delta)(t-s)} e^{\delta s} \mathrm{~d} s \\
& +\frac{\theta}{\lambda-\delta} e^{\delta \tau} \sup _{-\tau \leqslant s \leqslant t}\left\{\|u(x, s)\|_{L^{2}}^{2} e^{\delta s}\right\} .
\end{aligned}
$$

注意到 $g(t)=\sup _{-\tau \leqslant s \leqslant t}\left\{\|u(x, s)\|_{L^{2}}^{2} e^{\delta s}\right\}$ 是单调在 区间 $[-\tau,+\infty)$ 上不减函数, 对任意 $t>0$, 从而由式 (21) 可得

$$
\begin{aligned}
g(t)= & \sup _{-\tau \leqslant s \leqslant t}\left\{\|u(x, s)\|_{L^{2}}^{2} e^{\delta s}\right\} \\
\leqslant & \sup _{-\tau \leqslant s \leqslant 0}\left\{\|u(x, s)\|_{L^{2}}^{2} e^{\delta s}\right\} \\
& +\sup _{0 \leqslant s \leqslant t}\left\{\|u(x, s)\|_{L^{2}}^{2} e^{\delta s}\right\} \\
\leqslant & 2 \sup _{-\tau \leqslant s \leqslant 0}\left\{\|u(x, s)\|_{L^{2}}^{2} e^{\delta s}\right\} \\
& +\frac{\theta e^{\delta \tau}}{\lambda-\delta} g(t) .
\end{aligned}
$$

从而有

$$
g(t)=\sup _{-\tau \leqslant s \leqslant t}\left\{\|u(x, s)\|_{L^{2}}^{2} e^{\delta s}\right\} \leqslant \frac{2 g(0)}{f(\delta)} .
$$

将式 (23) 代入式 (21) 得

$$
\begin{aligned}
& \|u(x, t)\|_{L^{2}}^{2} e^{\delta t} \\
& \leqslant\|u(x, 0)\| e^{-(\lambda-\delta) t} \\
& \quad-\varepsilon \int_{0}^{t}\|\nabla(u(x, s))\|_{L^{2}}^{2} \\
& \quad e^{-(\lambda-\delta)(t-s)} e^{\delta s} \mathrm{~d} s+\frac{\theta e^{\delta \tau}}{\lambda-\delta} \frac{2 g(0)}{f(\delta)}
\end{aligned}
$$




$$
\begin{aligned}
\leqslant & \frac{2-f(\delta)}{f(\delta)} g(0)-\varepsilon \int_{0}^{t}\|\nabla(u(x, s))\|_{L^{2}}^{2} \\
& e^{-(\lambda-\delta)(t-s)} e^{\delta s} \mathrm{~d} s .
\end{aligned}
$$

因此, 对取定的 $T>0$, 当 $t>T$ 时,

$$
\begin{aligned}
\|u(x, t)\|_{L^{2}}^{2} e^{\delta t} & \\
\leqslant & \frac{2-f(\delta)}{f(\delta)} g(0) \\
& -\varepsilon \int_{0}^{t}\|\nabla(u(x, s))\|_{L^{2}}^{2} e^{-(\lambda-\delta)(t-s)} e^{\delta s} \mathrm{~d} s \\
\leqslant & \frac{2-f(\delta)}{f(\delta)} g(0) \\
& -\varepsilon T e^{-(\lambda-\delta) T}\|\nabla(u(x, s))\|_{L^{2}}^{2} e^{\eta \delta} .
\end{aligned}
$$

$\eta \in[t-T, t]$, 于是当 $t>T$ 时, 有

$\|u(x, t)\|_{L^{2}}^{2} e^{\delta t}+\varepsilon T e^{-(\lambda-\delta) T}\|\nabla(u(x, s))\|_{L^{2}}^{2} e^{\eta \delta}$

$\leqslant \frac{2-f(\delta)}{f(\delta)} g(0), \quad \eta \in[t-T, t]$.

注意到右端为常量, 可得 $\lim _{t \rightarrow \infty}\|u(x, t)\|_{L^{2}}^{2} e^{\delta t}=0$, 而且有

$$
\|\nabla(u(x, s))\|_{L^{2}}^{2} e^{\eta \delta} \leqslant \frac{1}{\varepsilon T} e^{(\lambda-\delta) T} \frac{2-f(\delta)}{f(\delta)} g(0) .
$$

从而

$$
\begin{aligned}
& \lim _{t \rightarrow \infty}\|\nabla(u(x, s))\|_{L^{2}}^{2} e^{\frac{\delta}{2} \eta} \\
& =\lim _{\eta \rightarrow \infty}\|\nabla(u(x, s))\|_{L^{2}}^{2} e^{\frac{\delta}{2} \eta} \\
& \leqslant \lim _{\eta \rightarrow \infty} \frac{1}{\varepsilon T} e^{(\lambda-\delta) T} \frac{2-f(\delta)}{f(\delta)} g(0) e^{-\frac{\delta}{2} \eta}=0 .
\end{aligned}
$$

\section{于是, 我们得到}

$$
\lim _{t \rightarrow \infty}\|u(x, t)\|_{L^{2}}^{2} e^{\delta t}=\lim _{t \rightarrow \infty}\|\nabla(u(x, s))\|_{L^{2}}^{2} e^{\frac{\delta}{2} t}=0 .
$$

所以, 系统 (1) 的平衡点是 $W^{1,2}(\Omega)$-指数渐近稳定的.

推论 1 对于系统 (1), 若存在正数 $\varepsilon, \alpha$, 并选取 $\gamma=\alpha \sum_{j=1}^{n} l_{j}^{2}$, 使得

(i) $(2 \tilde{D}-\varepsilon I)>0$,

(ii) $\lambda=-\lambda_{\max }\left(-2 C+B B^{\mathrm{T}}+A A^{\mathrm{T}}\right)+\frac{m}{l^{2}}$. $\lambda_{\min }(2 \tilde{D}-\varepsilon I)>0$,

(iii) $\lambda>\frac{\gamma}{\alpha}$.

则有系统 $(1)$ 是全局 $W^{1,2}(\Omega)$ - 指数渐近稳定的.

\section{2 变时滞系统的稳定性}

考虑下列系统

$$
\frac{\partial \bar{u}_{i}}{\partial t}=\sum_{k=1}^{m} \frac{\partial}{\partial x_{k}}\left(D_{i k} \frac{\partial \bar{u}_{i}}{\partial x_{k}}\right)-c_{i} \bar{u}_{i}(t, x)
$$

$$
\begin{aligned}
& +\sum_{j=1}^{n} a_{i j} \tilde{f}_{j}\left(\bar{u}_{j}(t, x)\right) \\
& +\sum_{j=1}^{n} b_{i j} \tilde{f}_{j}\left(\bar{u}_{j}\left(t-\tau_{j}(t), x\right)\right) \\
& +I_{i}, x \in \Omega, \quad i=1, \ldots, n .
\end{aligned}
$$

满足初边值条件

$$
\begin{aligned}
& \frac{\partial \bar{u}_{i}}{\partial n}=0, t \geqslant 0, x \in \partial \Omega, \quad i=1, \ldots, n . \\
& \bar{u}_{i}(s, x)=\bar{\varphi}_{i}(s, x) \\
& -\tau \leqslant s \leqslant 0, i=1, \ldots, n .
\end{aligned}
$$

其中符号定义与模型 $(1)$ 相同, 而 $\tau_{j}(t)$ 是变时滞.

定理 2 若

$$
\begin{aligned}
\lambda= & -\lambda_{\max }\left[2 C+\sum_{j=1}^{n} B^{\mathrm{T}} P_{2 k} B+L^{\mathrm{T}} P_{1}^{-1} L+A^{\mathrm{T}} P_{1} A\right] \\
& +\frac{m}{l^{2}} \lambda_{\min }(2 \tilde{D}-\varepsilon I)>0 \\
\lambda- & \frac{m}{l^{2}}-n \max _{1 \leqslant k \leqslant n}\left\|L^{\mathrm{T}} P_{2 k}^{-1} L\right\|>0,
\end{aligned}
$$

则系统 $(30)$ 是 $W^{1,2}(\Omega)$-指数渐近稳定的.

证明 作辅助函数

$$
\begin{aligned}
V(x, t)= & \alpha u^{\mathrm{T}}(x, t) u(x, t)+\beta \int_{0}^{t} u^{\mathrm{T}}(x, s) u(x, s) \mathrm{d} s \\
& -\gamma \sum_{k=1}^{n} \int_{0}^{t} u^{\mathrm{T}}\left(x, s-\tau_{k}(s)\right) u\left(x, s-\tau_{k}(s)\right) \mathrm{d} s .
\end{aligned}
$$

其中 $\alpha, \beta, \gamma$ 为待定的常数.

其证明方法与定理 1 相同, 此处省略.

\section{3 分布时滞系统的稳定性}

考虑系统

$$
\begin{aligned}
\frac{\partial \bar{u}_{i}}{\partial t}= & \sum_{k=1}^{m} \frac{\partial}{\partial x_{k}}\left(D_{i k} \frac{\partial \bar{u}_{i}}{\partial x_{k}}\right)-c_{i} \bar{u}_{i}(x, t) \\
& +\sum_{j=1}^{n} a_{i j} \tilde{f}_{j}\left(\bar{u}_{j}(x, t)\right) \\
& +\sum_{j=1}^{n} b_{i j} \tilde{f}_{j}\left(\int_{-\infty}^{t} k_{j}(s)\left(\bar{u}_{j}(x, s)\right) \mathrm{d} s+I_{i},\right. \\
& x \in \Omega, \quad i=1, \ldots, n .
\end{aligned}
$$

满足初边值条件

$$
\begin{aligned}
& \frac{\partial \bar{u}_{i}}{\partial n}=0, t \geqslant 0, x \in \partial \Omega, \quad i=1, \ldots, n . \\
& \bar{u}_{i}(x, s)=\bar{\varphi}_{i}(x, s), \\
& -\infty \leqslant s \leqslant 0, \quad i=1, \ldots, n .
\end{aligned}
$$


假设模型 $(31)$ 激活函数 $\tilde{f}_{j}(u, t)(j=1, \ldots, n)$ 满 足假设条件 (H1), 并且 $\Omega=\left\{x=\left(x_{1}, \ldots, x_{m}\right),\left|x_{i}\right|<\right.$ $h\}$ 与模型 (1) 类似的变换, 将 (1) 式可转化为下列矩 阵形式:

$$
\begin{aligned}
\frac{\partial u}{\partial t}= & D \Delta u(x, t)-C u(x, t)+A f(u(x, t)) \\
& +B f\left(\int_{-\infty}^{t} k(t-s) u(x, t-s) \mathrm{d} s\right) .
\end{aligned}
$$

定义 $3^{[29]}$ 若对任意的 $\varepsilon>0$, 存在 $\delta(\varepsilon)>0$ 及 $N_{1}>0$, 使得当 $M_{1}=\sup _{-\infty \leqslant s \leqslant 0} \int_{\Omega}\|\varphi(x, s)\|_{L^{2}}^{2} \mathrm{~d} x<$ $\delta(\varepsilon)$ 时, 有

$$
\|u(x, t)\|_{L^{2}} \leqslant \varepsilon,\|\nabla u(x, t)\|_{L^{2}} \leqslant N_{1} \varepsilon, \quad t \in \mathbb{R}^{+},
$$

则称 (31) 式的零解是 $X^{1,2}(\Omega)$-稳定的, 若还有 $\lim _{t \rightarrow \infty}\|u(x, t)\|_{L^{2}}=\lim _{t \rightarrow \infty}\|\nabla u(x, t)\|_{L^{2}}=0$, 则称 (31) 式的零解是 $X^{1,2}(\Omega)$-渐近稳定的, 其中 $\|u(x, t)\|_{L^{2}}=\left\{\int_{\Omega}|\cdot|^{2} \mathrm{~d} x\right\}^{\frac{1}{2}}$.

定理 3 若式 $(31)$ 满足 $\int_{-\infty}^{t} k_{j}(t-s) \mathrm{d} s=$ $1,\|k(t-s)\| \leqslant e^{-\delta(t-s)}$, 则当 $b<\lambda \delta$ 时, (31) 式的 零解是 $X^{1,2}(\Omega)$ - 渐近稳定的.

其中 $b=\|B\|\|L\|, \lambda=-\lambda_{\max }\left[\frac{1}{2}\left(-2 C+A P_{1} A^{\mathrm{T}}\right.\right.$ $\left.\left.+L^{\mathrm{T}} P_{1}^{-1} L\right)\right]+\frac{\lambda_{\min }(\tilde{D})}{h^{2}}$, 其中 $\tilde{D}$ 的定义与定理 1 相同.

证明 由 $b<\lambda \delta$, 必存在充分小的 $\varepsilon, \sigma$ 使得

$b\left(\frac{1}{\delta}+\frac{1}{\delta-2 \sigma}\right) / 2\left(\lambda-\varepsilon h^{-2}-\sigma\right)<1$.

用 $u^{\mathrm{T}}(x, t)$ 乘以 $(32)$ 的两端, 并关于 $x$ 积分有

$$
\begin{aligned}
& \frac{\partial}{\partial t} \int_{\Omega} u^{\mathrm{T}}(x, t) u(x, t) \mathrm{d} x \\
& =2 \sum_{i=1}^{n} \int_{\Omega} u_{i}(x, t) \sum_{k=1}^{m} \frac{\partial}{\partial x_{k}}\left(D_{i k} \frac{\partial u_{i}}{\partial x_{k}}\right) \\
& \quad-2 C \int_{\Omega} u^{\mathrm{T}}(x, t) u(x, t) \mathrm{d} x \\
& \quad+2 \int_{\Omega} u^{\mathrm{T}}(x, t) A f(u(x, t)) \mathrm{d} x \\
& \quad+2 \int_{\Omega} u^{\mathrm{T}}(x, t) B f\left(\int_{-\infty}^{t}(u(x, s)) \mathrm{d} s\right) \mathrm{d} x .
\end{aligned}
$$

由定理 1 的证明过程类似可得

$$
\begin{aligned}
& \int_{\Omega} \nabla u(x, t)^{\mathrm{T}} D \nabla u(x, t) \mathrm{d} x \\
& \geqslant \frac{\lambda_{\min }(\tilde{D})}{h^{2}} \int_{\Omega} u(x, t)^{\mathrm{T}} u(x, t) \mathrm{d} x .
\end{aligned}
$$

所以有

$$
\frac{\mathrm{d}}{\mathrm{d} t} \int_{\Omega} u^{\mathrm{T}}(x, t) u(x, t) \mathrm{d} x
$$

$$
\begin{aligned}
\leqslant & -\int_{\Omega} \nabla u(x, t)^{\mathrm{T}} D \nabla u(x, t) \mathrm{d} x \\
& -2 C \int_{\Omega} u^{\mathrm{T}}(x, t) u(x, t) \mathrm{d} x \\
& +\int_{\Omega} u^{\mathrm{T}}(x, t) A P_{1} A^{\mathrm{T}} u(t, x) \mathrm{d} x \\
& +\int_{\Omega} u^{\mathrm{T}}(x, t) L^{\mathrm{T}} P_{1}^{-1} L u(x, t) \mathrm{d} x \\
& +2 \int_{\Omega} u^{\mathrm{T}}(x, t) B \\
& \cdot f\left(\int_{-\infty}^{t} k_{j}(s)(u(x, s)) \mathrm{d} s\right) \mathrm{d} x .
\end{aligned}
$$

对上述的 $\varepsilon$ 有,

$$
\begin{aligned}
& -\int_{\Omega} \nabla u(x, t)^{\mathrm{T}} D \nabla u(x, t) \mathrm{d} x \\
& \leqslant-\varepsilon \int_{\Omega} \nabla u(x, t)^{\mathrm{T}} \nabla u(x, t) \mathrm{d} x \\
& -\left(\frac{\lambda_{\min }(\tilde{D})}{h^{2}}-\varepsilon\right) \int_{\Omega} u(x, t)^{\mathrm{T}} u(x, t) \mathrm{d} x .
\end{aligned}
$$

故 (36) 化为

$$
\begin{aligned}
\frac{\mathrm{d}}{\mathrm{d} t} & \int_{\Omega}\|u(x, t)\|^{2} \mathrm{~d} x \\
\leqslant & -2 \varepsilon \int_{\Omega} \nabla u(x, t)^{\mathrm{T}} \nabla u(x, t) \mathrm{d} x \\
& -\frac{2}{h^{2}}\left(\lambda_{\min }(\tilde{D})-\varepsilon\right) \int_{\Omega} u(x, t)^{\mathrm{T}} u(x, t) \mathrm{d} x \\
& +\int_{\Omega} u^{\mathrm{T}}(x, t)\left(-2 C+A P_{1} A^{\mathrm{T}}\right. \\
& \left.+L^{\mathrm{T}} P_{1}^{-1} L\right) u(x, t) \mathrm{d} x \\
& +2\|B\|\|L\| \int_{\Omega} \int_{-\infty}^{t}\|k(t-s)\| \\
& \|u(x, t)\| \cdot\|u(x, s)\| \mathrm{d} s \mathrm{~d} x \\
\leqslant & -2 \varepsilon \int_{\Omega} \nabla u(x, t)^{\mathrm{T}} \nabla u(x, t) \mathrm{d} x \\
& -\frac{2}{h^{2}}\left(\lambda_{\min }(D)-\varepsilon\right) \int_{\Omega} u(x, t)^{\mathrm{T}} u(x, t) \mathrm{d} x \\
& +\int_{\Omega} u^{\mathrm{T}}(x, t)\left(-2 C+A P_{1} A^{\mathrm{T}}\right. \\
& \left.+L^{\mathrm{T}} P_{1}^{-1} L\right) u(x, t) \mathrm{d} x \\
& +2 b \int_{\Omega}^{t} \int_{-\infty}^{t}\|k(t-s)\|\|u(x, t)\|\|u(x, s)\| \mathrm{d} s \mathrm{~d} x \\
\leqslant & -2 \varepsilon \int_{\Omega}(\nabla u(x, t))^{2} \mathrm{~d} x \\
& -2\left(\lambda-\frac{\varepsilon}{h^{2}}\right) \int_{\Omega}\|u(x, t)\|^{2} \mathrm{~d} x
\end{aligned}
$$




$$
\begin{aligned}
& +\frac{b}{\delta} \int_{\Omega}\|u(x, t)\|^{2} \mathrm{~d} x \\
& +b \int_{\Omega} \int_{0}^{\infty} e^{-\delta s}\|u(x, t-\theta)\|^{2} \mathrm{~d} \theta \mathrm{d} x
\end{aligned}
$$

所以, 有

$$
\begin{aligned}
& \int_{\Omega}\|u(x, t)\|^{2} \mathrm{~d} x \\
& \leqslant M_{1} e^{-2\left(\lambda-\varepsilon h^{-2}\right)(t-s)} \\
& \quad-2 \varepsilon \sum_{i=1}^{n} \int_{0}^{t} e^{-2\left(\left(\lambda-\varepsilon h^{-2}\right)(t-s)\right.} \int_{\Omega}\left(\nabla u_{i}(x, t)\right)^{2} \mathrm{~d} x \\
& \quad+\frac{b}{\delta} \int_{0}^{t} e^{-2\left(\lambda-\varepsilon h^{-2}\right)(t-s)} \int_{\Omega}\|u(x, s)\|^{2} \mathrm{~d} x \mathrm{~d} s \\
& \quad+\int_{0}^{t} e^{-2\left(\lambda-\varepsilon h^{-2}\right)(t-s)} \int_{\Omega} \int_{0}^{\infty} b e^{-\delta \theta} \\
& \|u(x, s-\theta)\|^{2} \mathrm{~d} \theta \mathrm{d} x \mathrm{~d} s .
\end{aligned}
$$

其中, $M_{1}=\sup _{-\infty \leqslant s \leqslant 0} \int_{\Omega}\|\varphi(x, s)\|^{2} \mathrm{~d} x$. 对 $\sigma>0$, 则有

$$
\begin{aligned}
& e^{2 \sigma t} \int_{\Omega}\|u(x, t)\|^{2} \mathrm{~d} x \\
& \leqslant M_{1}-2 \varepsilon \sum_{i=1}^{n} \int_{0}^{t} e^{-2\left(\left(\lambda-\varepsilon h^{-2}-\sigma\right)(t-s)\right.} \\
& \int_{\Omega}\left(\nabla_{i} u(x, t)\right)^{2} \mathrm{~d} x \\
& +\frac{b}{2\left(\lambda-\varepsilon h^{-2}-\sigma\right)}\left(\frac{1}{\delta}+\frac{1}{\delta-2 \sigma}\right) \\
& \sup _{-\infty \leqslant s \leqslant t}\left\{\int_{\Omega} e^{2 \sigma s}\|u(x, s)\|^{2} \mathrm{~d} x\right\} .
\end{aligned}
$$

注意到右端是单调不减的, 记 $p(t)=$ $\sup _{-\infty \leqslant \theta \leqslant t}\left\{\int_{\Omega} e^{2 \sigma \theta}\|u(x, \theta)\|^{2} \mathrm{~d} x\right\}$, 则

$$
\begin{aligned}
& \sup _{0 \leqslant s \leqslant t}\left\{e^{2 \sigma t} \int_{\Omega}\|u(x, t)\|^{2} \mathrm{~d} x\right\} \\
& \leqslant M_{1}+\frac{b}{2\left(\lambda-\varepsilon h^{-2}-\sigma\right)}\left(\frac{1}{\delta}+\frac{1}{\delta-2 \sigma}\right) p(t) .
\end{aligned}
$$

而

$$
\begin{aligned}
& \sup _{-\infty \leqslant s \leqslant t}\left\{\int_{\Omega} e^{2 \sigma s}\|u(x, s)\|^{2} \mathrm{~d} x\right\} \\
& \leqslant M_{1}+\sup _{0 \leqslant s \leqslant t}\left\{\int_{\Omega} e^{2 \sigma s}\|u(x, s)\|^{2} \mathrm{~d} x\right\} .
\end{aligned}
$$

故有

$$
p(t) \leqslant 2 M_{1}+\frac{b}{2\left(\lambda-\varepsilon h^{-2}-\sigma\right)}\left(\frac{1}{\delta}+\frac{1}{\delta-2 \sigma}\right) p(t) .
$$

记 $1-\frac{b}{2\left(\lambda-\varepsilon h^{-2}-\sigma\right)}\left(\frac{1}{\delta}+\frac{1}{\delta-2 \sigma}\right)=d$, 所以有

$$
p(t) \leqslant 2 M_{1} / d \text {. }
$$

将上式代入 (40) 式, 对 $t>a>0$ 有

$$
\begin{aligned}
& e^{2 \sigma t} \int_{\Omega}\|u(x, t)\|^{2} \mathrm{~d} x \\
& \leqslant M_{1}\left[1+\frac{2}{d}(1-d)\right] \\
& -2 \varepsilon \int_{t-a}^{t} e^{-2\left(\left(\lambda-\varepsilon h^{-2}-\sigma\right)(t-s)\right.} \\
& \sum_{i=1}^{n}\left\{e^{2 \sigma s} \int_{\Omega}\left\|\nabla u_{i}(x, t)\right\|^{2}\right\} \mathrm{d} x .
\end{aligned}
$$

注意到

$$
\begin{aligned}
& -2 \varepsilon \int_{t-a}^{t} e^{-2\left(\left(\lambda-\varepsilon h^{-2}-\sigma\right)(t-s)\right.} \\
& \sum_{i=1}^{n}\left\{e^{2 \sigma s} \int_{\Omega}\left\|\nabla u_{i}(x, t)\right\|^{2}\right\} \mathrm{d} x \\
& \leqslant-2 \varepsilon a e^{-2\left(\lambda-\varepsilon h^{-2}-\sigma\right) a} \\
& \sum_{i=1}^{n} \int_{\Omega}\left\{e^{2 \sigma \eta}\left\|\nabla u_{i}(x, \eta)\right\|^{2}\right\} \mathrm{d} x, \eta \in[t-a, t] .
\end{aligned}
$$

由 (45),(46) 式

$$
\begin{gathered}
e^{2 \sigma t} \int_{\Omega}\|u(x, t)\|^{2} \mathrm{~d} x+2 \varepsilon a e^{-2\left(\lambda-\varepsilon h^{-2}-\sigma\right) a} \\
\sum_{i=1}^{n} \int_{\Omega}\left\{e^{2 \sigma \eta}\left\|\nabla u_{i}(x, \eta)\right\|^{2}\right\} \mathrm{d} x \leqslant M_{1} \frac{2-d}{d} .
\end{gathered}
$$

因上式右端与 $t$ 无关, 对 $t>a$ 有

$$
\begin{aligned}
Q(t)= & \sup _{-\infty \leqslant s \leqslant t}\left\{e^{2 \sigma s} \int_{\Omega}\|u(x, s)\|^{2} \mathrm{~d} x\right\} \\
& +2 \varepsilon a e^{-2\left(\lambda-\varepsilon h^{-2}-\sigma\right) a} \\
& \sum_{i=1}^{n} \int_{\Omega-\infty \leqslant s \leqslant t} \sup _{-\infty}\left\{e^{2 \sigma s}\left\|\nabla u_{i}(x, s)\right\|^{2}\right\} \mathrm{d} x \\
\leqslant & M_{1} \frac{2-d}{d} .
\end{aligned}
$$

从而

$$
\begin{aligned}
& \int_{\Omega}\|u(x, t)\|^{2} \mathrm{~d} x+2 \varepsilon a e^{-2\left(\lambda-\varepsilon h^{-2}-\sigma\right) a} \\
& \sum_{i=1}^{n} \int_{\Omega}\left\|\nabla u_{i}(x, t)\right\|^{2} \mathrm{~d} x \\
& \leqslant Q(t) e^{-2 \sigma t} \leqslant M_{1} \frac{2-d}{d} e^{-2 \sigma t} .
\end{aligned}
$$

由 (49) 式便可推得 (31) 式的零解是 $X^{1,2}(\Omega)$ - 渐近 稳定的.

\section{3 举例说明}

对系统 (1), 设 $f_{1}=f_{2}=\frac{1}{2}(|u+1|-|u-1|)$, 从 
而 $L=I, l=2, m=3$, 取 $\alpha=0.5$, 所以

$$
\begin{gathered}
\gamma=0.5 \quad D=\left(\begin{array}{ll}
1 & 0 \\
0 & 2
\end{array}\right), \\
\varepsilon=0.5, \quad C=\left(\begin{array}{ll}
3 & 0 \\
0 & 2
\end{array}\right), \\
B=\left(\begin{array}{cc}
-1 & 0 \\
0 & 0.5
\end{array}\right), \quad A=\left(\begin{array}{cc}
1 & 0 \\
0 & -0.5
\end{array}\right),
\end{gathered}
$$

显然, 推论 1 的条件 (i) 满足, 且 $-\lambda=\lambda_{\max }(-2 C+$ $\left.B P_{2} B^{\mathrm{T}}+A P_{1} A^{\mathrm{T}}\right)-\frac{m}{l^{2}} \cdot \lambda_{\min }(2 D-\varepsilon I)=-\frac{25}{8}<0$, 故推论 1 的条件 (ii) 与 (iii) 也满足. 所以根据推论
1 得系统 (1) 是全局 $W^{1,2}(\Omega)$-指数渐近稳定的.

\section{4 结论}

本文针对一类具反应扩散项的时滞细胞神经网 络模型, 利用 Poincare 不等式与散度定理, 得到了系 统关于 $W^{1,2}(\Omega)$-指数稳定性与 $X^{1,2}(\Omega)$-渐近稳定性 的条件, 这些稳定性条件与反应扩散算子有关. 这说 明系统的反应扩散算子在系统的稳定性中起着作用. 这是本文与已往研究含反应扩散项的神经网络模型 的稳定性条件的一个显著的区别. 最后, 我们通过算 例说明我们所得结论的可行性.

\section{参考文献}

1 Lu H T, Shen R M, Chung F L. Absolute exponential stability of a class of recurrent neural networks with multiple and variable delays. Theor Comput Sci, 2005, 344(2-3), 103-119

2 Yuan K, Cao J D. An analysis of global Asymptotic stability of delayed Cohen-Grossberg neural networks via nonsmooth analysis. IEEE Trans Circuits-I: Regular Papers, 2005, 52(9): 1854-1861

3 Tu F H, Liao X F, Zhang W. Delay-dependent asymptotic stability of a two-neural system with different time delays. Chaos, Soliton Fract, 2006, 28(2): 437-447

4 Sabri A, Vedat T. Global asymptotic stability analysis of bidirectional associative memory neural networks with constant time delays. Neurocomputing, 2005, 68(10): 161-176

5 Liao X X, Wang J, Zeng Z G. Global asymptotic stability and global exponential stability of delayed cellular neural networks. IEEE Trans Circuits-II: Express Briefs, 2005, 52(7): 403-409

6 Zhang Q, Wei X P, Xu J. An improved result for complete stability of delayed cellular neural networks. Automatica, 2005, 41(2): $333-337$

7 Cao J D, Daniel W C H. A general framework for global asymptotic stability analysis of delayed neural networks based on LMI approach. Chaos, Soliton Fract, 2005, 24(5): 1317-1329

8 Zhang Y. Global exponential convergence of recurrent neural networks with variable delays. Theor Comput Sci, 2004, 312(2-3): $281-293$

9 鲁丽, 张继业, 杨翊仁. 一类时滞 Hopfield 神经网络系统的全局指数稳定. 电子学报, 2002, 30(10): 1431-1434

10 Li X M, Huang L H, Zhu H Y. Global stability of cellular neural networks with constant and variable delays. Nonlinear Anal-Theor, 2003, 53(3-4): 319-333

11 刘妹琴. 离散时滞标准神经网络模型及其应用, 中国科学 E 辑: 技术科学, 2005, 35(10): 1031-1048

12 廖晓昕, 杨叔子, 程时杰等. 具有反应扩散的广义神经网络的稳定性. 中国科学 E 辑: 技术科学, 2002, 32(1): 87-94

13 王林山, 徐道义. 变时滞反应扩散 Hopfield 神经网络的全局指数稳定性. 中国科学 E 辑: 技术科学, 2003, 33(6): 488-495

14 Liang J L, Cao J D. Global exponential stability of reaction-diffusion recurrent neural networks with time-varying delays. Phys Lett A, 2004, 314(5-6): 434-442

15 Son Q K, Zhao Z J, Li Y M. Global exponential stability of BAM neural networks with distributed delays and reaction-diffusion terms. Phys Lett A, 2005, 335(2-3): 213-225

16 戴志娟, 孙建华. 一类具有连续分布延时的随机反应扩散的 Hopfield 神经网络的收玫动力学行为. 南京大学学报 (数学半 年刊), 2005, 22(2): 197-211

17 丁桶, 赵洪涌. 带反应扩散和分布时滞的神经网络的稳定性. 新疆师范大学学报 (自然科学版), 2005, 24(3): 9-11

18 郑伟范, 张继业. 具有反应扩散的神经网络的稳定性分析. 西南交通大学学报, 2004, 39(1): 117-120

19 Zhao Z J, Song Q K. Exponential periodicity and stability of neural networks with reaction-diffusion terms and both variable and unbounded delays. Comput Math Appl, 2006, 51(3-4): 475-486 
20 Qiu J L. Exponential stability of impulsive neural networks with time-varying delays and reaction-diffusion terms. Neurocomputing, 2007, 70(4-6): 1102-1108

21 Zhou Q H, Wan L, Sun J H. Exponential stability of reaction-diffusion generalized Cohen-Grossberg neural networks with time-varying delays. Chaos, Soliton Fract, 2007, 32(5): 1713-1719

22 Cui B T, Lou X Y. Global asymptotic stability of BAM neural networks with distributed delays and reaction-diffusion terms. Chaos, Soliton Fract, 2006, 27(5): 1347-1354

23 Song Q K, Cao J D. Global exponential robust stability of Cohen-Grossberg neural network with time-varying delays and reaction-diffusion terms. J Franklin Inst, 2006, 343(7): 705-719

24 Wang L S, Gao Y Y. Global exponential robust stability of reaction-diffusion interval neural networks with time-varying delays. Phys Lett A, 2006, 350(5-6): 342-348

25 Song Q K, Cao J D. Global exponential stability and existence of periodic solutions in BAM networks with delays and reaction-diffusion terms. Chaos, Soliton Fract, 2005, 23(2): 421-430

26 Lou X Y, Cui T, Wu W. On global exponential stability and existence of periodic solutions for BAM neural networks with distributed delays and reaction-diffusion terms. Chaos, Soliton Fract, 2008, 36(4): 1044-1054

27 Song Q K, Cao J D, Zhao Z J. Periodic solutions and its exponential stability of reaction-diffusion recurrent neural networks with continuously distributed delays. Nonlinear Anal-Real, 2006, 7(1): 65-80

28 Allegretto W, Papini D. Stability for delayed reaction-diffusion neural networks. Phys Lett A, 2007, 360(6): 669-680

29 Sanchez E N, Perez J P. Input-to-state stability analysis for dynamic NN. IEEE Trans Circuits-I, 1999, 46(11): 1395-1398

30 刘永清, 谢胜利. 滞后分布参数系统的稳定与变结构控制. 广州: 华南理工大学出版社, 1998. 12-14

31 Robert C. McOwen Partial Differential Equations: Methods and Applications. Upper Saddle Rive: Prentice Hall, Inc, 1996. $163-164$ 